\title{
ON THE POSITIVITY OF RIEMANN-STIELTJES INTEGRALS
}

\author{
JANI LUKKARINEN AND MIKKO S. PAKKANEN
}

\begin{abstract}
We study the question, whether a Riemann-Stieltjes integral of a positive continuous function with respect to a non-negative function of bounded variation is positive.
\end{abstract}

\section{INTRODUCTION}

Let $f:[a, b] \rightarrow \mathbb{R}$ be a continuous function and $g:[a, b] \rightarrow \mathbb{R}$ a function of bounded variation. It is a classical result that for such $f$ and $g$, and for any $y \in(a, b]$, the Riemann-Stieltjes integral

$$
\int_{a}^{y} f(x) \mathrm{d} g(x),
$$

exists (see, e.g., [7, pp. 316-317]). While the basic properties of Riemann-Stieltjes integrals (and related Lebesgue-Stieltjes integrals) are covered in classical textbooks on real analysis and integration $[4,5,7,8]^{1}$, the following simple question is not addressed in them.

Question 1. If $f$ is positive, $g$ is non-negative, non-vanishing, and satisfies $g(a)=0$, can we then select the upper limit of integration y so that the integral (1) is positive?

The answer to the question is obviously yes for Riemann integrals $(g(x)=x-a)$, which could help to explain why it has been overlooked so far. In fact, after an extensive search of the literature, we believe that Question 1 has not been answered before in the full generality. (We shall comment later, in Remark 3, why the present generality may be relevant in applications.) The only reference known to us is a note by Satyanarayana [9] where an affirmative answer is proven under a slightly different set of assumptions, most importantly, assuming that $g$ is non-decreasing.

Let us stress that positive will here always mean strictly positive. In particular, since $f$ is continuous, under our assumptions $0<\min f \leqslant \max f<\infty$. Recall that, since $g$ is of bounded variation, there exist non-decreasing functions $g^{+}$and $g^{-}$such that $g=g^{+}-g^{-}$. Therefore, finite $\lim _{x \rightarrow y^{+}} g(x)$ and $\lim _{x \rightarrow y^{-}} g(x)$ exist for any $y \in[a, b]$

Date: June 4, 2013.

2000 Mathematics Subject Classification. Primary 26A42; Secondary 26A45.

Key words and phrases. Riemann-Stieltjes integral, positivity, function of bounded variation, Grönwall's inequality.

${ }^{1}$ Protter and Morrey [7] have a particularly comprehensive account of Riemann-Stieltjes integrals. 
(apart from $\lim _{x \rightarrow a^{-}} g(x)$ and $\lim _{x \rightarrow b^{+}} g(x)$, obviously). Moreover, the integral (1) is equal to

$$
\int_{a}^{y} f(x) \mathrm{d} g^{+}(x)-\int_{a}^{y} f(x) \mathrm{d} g^{-}(x) .
$$

Since $g(a)=0$, we may assume that $g^{+}$and $g^{-}$are non-negative and satisfy $g^{ \pm}(a)=0$.

We may now distinguish two special cases, where it is evident that the answer to Question 1 is yes.

(1) If $\lim _{x \rightarrow x_{\mathrm{L}}^{+}} g(x)>0$, where $x_{\mathrm{L}}:=\inf \{x: g(x)>0\}$, then there exists $\varepsilon>0$ such that (1) is positive for $y=x_{\mathrm{L}}+\varepsilon$.

(2) If $g^{+}(y)>0$ and $g^{-}(y)=0$, then (1) is at least $g^{+}(y) \min _{x \in[a, y]} f(x)>0$.

The first item follows from the elementary lower bound, valid for $0<\varepsilon \leq b-x_{\mathrm{L}}$,

$$
\int_{a}^{x_{\mathrm{L}}+\varepsilon} f(x) \mathrm{d} g(x) \geqslant g^{+}\left(x_{\mathrm{L}}+\varepsilon\right) \min _{a, x_{\mathrm{L}}-\varepsilon \leq x \leq x_{\mathrm{L}}+\varepsilon} f(x)-g^{-}\left(x_{\mathrm{L}}+\varepsilon\right) \max _{a, x_{\mathrm{L}}-\varepsilon \leq x \leq x_{\mathrm{L}}+\varepsilon} f(x),
$$

whereas the second item is a straightforward consequence of $g^{ \pm}(a)=0$.

In general, the integral (1) is positive if and only if

$$
\int_{a}^{y} f(x) \mathrm{d} g^{+}(x)>\int_{a}^{y} f(x) \mathrm{d} g^{-}(x) .
$$

Obviously, the condition $g \geqslant 0$ is equivalent to $g^{+} \geqslant g^{-}$, and we have $g(x)>0$ if and only if $g^{+}(x)>g^{-}(x)$. It would be tempting to conjecture that, as a continuous function, $f$ is "nearly constant" in some neighborhood of $x_{\mathrm{L}}$ and, hence, that the inequality (2) ought to hold for $y=x_{\mathrm{L}}+\varepsilon$ with some "small" $\varepsilon>0$, suggesting an affirmative answer to Question 1 in general.

Remark 1. The proviso $g(a)=0$ may seem superfluous as the value of the integral (1) does not depend on $g(a)$. However, together with the condition $g \geqslant 0$ it constrains the behavior of $g$ near $x_{\mathrm{L}}$, which is a key part of the formulation of Question 1. It should also be stressed that the continuity of $f$ is equally important-aside from the possible non-existence of the integral, there is no reason to expect the answer to Question 1 to be yes when $f$ fails to be continuous.

\section{Negative Answer to Question 1}

Unfortunately, the heuristic above is too simple-minded since mere continuity does not restrict the fine properties of the integrand $f$ and leaves it with enough room to vary "too much" for our purposes. Indeed, the general answer to Question 1 is no. We show that for any $f$ that exhibits "enough" variation, there exists a suitable $g$ such that the integral (1) is less than zero for all $y \in(a, b]$.

Theorem 1. Let $f:[a, b] \rightarrow(0, \infty)$ be a continuous function. Suppose that there exist two sequences $\left(\underline{x}_{n}\right)$ and $\left(\bar{x}_{n}\right)$ with

$$
a<\cdots<\underline{x}_{n}<\bar{x}_{n}<\cdots<\underline{x}_{2}<\bar{x}_{2}<\underline{x}_{1}<\bar{x}_{1} \leqslant b \quad \text { and } \quad \lim _{n \rightarrow \infty} \bar{x}_{n}=a
$$


such that for some $\alpha>0$ and $\gamma \in(0,1)$,

$$
f\left(\bar{x}_{n}\right)-f\left(\underline{x}_{n}\right) \geqslant \alpha n^{-\gamma} \text { for all } n \in \mathbb{N} .
$$

Then, there exists a function $g:[a, b] \rightarrow[0, \infty)$ of bounded variation such that $g(a)=0$ and

$$
\int_{a}^{y} f(x) \mathrm{d} g(x)<0 \quad \text { for all } y \in(a, b] .
$$

Proof. Let $\beta>1$ and consider $h:[a, b] \rightarrow[0, \infty)$ defined by

$$
h(x):=\sum_{n \in \mathbb{N}} n^{-\beta} \chi_{\left[\underline{x}_{n}, \bar{x}_{n}\right)}(x),
$$

where $\chi_{E}$ denotes the characteristic function of a set $E$. (Figure 1 illustrates the definition for Example 1 below.) This is, by construction, a function of bounded variation such that $h(a)=0$. We have for any $n \in \mathbb{N}$,

$$
\int_{a}^{\underline{x}_{n}} f(x) \mathrm{d} h(x)=n^{-\beta} f\left(\underline{x}_{n}\right)-\sum_{k=n+1}^{\infty} k^{-\beta}\left(f\left(\bar{x}_{k}\right)-f\left(\underline{x}_{k}\right)\right),
$$

where

$$
\begin{aligned}
\sum_{k=n+1}^{\infty} k^{-\beta}\left(f\left(\bar{x}_{k}\right)-f\left(\underline{x}_{k}\right)\right) & \geqslant \alpha \sum_{k=n+1}^{\infty} k^{-(\beta+\gamma)} \\
& \geqslant \alpha \int_{n+2}^{\infty} x^{-(\beta+\gamma)} \mathrm{d} x \\
& =\frac{\alpha}{\beta+\gamma-1}(n+2)^{-(\beta+\gamma-1)}
\end{aligned}
$$

Since $\gamma<1$ and $\sup _{n \in \mathbb{N}} f\left(\underline{x}_{n}\right)<\infty$, there exists $n_{0} \in \mathbb{N}$ such that

$$
\int_{a}^{\underline{x}_{n}} f(x) \mathrm{d} h(x)<0 \quad \text { for all } n \geqslant n_{0} .
$$

We may also note that for all $n \geqslant n_{0}$, with $n \geqslant 2$, and $y \in\left(\underline{x}_{n}, \underline{x}_{n-1}\right)$,

$$
\int_{a}^{y} f(x) \mathrm{d} h(x) \leqslant \int_{a}^{\underline{x}_{n}} f(x) \mathrm{d} h(x)<0 .
$$

Thus, defining $g:=h \chi_{\left[a, \bar{x}_{n_{0}}\right)}$ yields a function with all the properties stated in the theorem.

Example 1. Function $f:[0,1] \rightarrow(0, \infty)$ given by

$$
f(x):= \begin{cases}x^{\gamma} \sin (1 / x)+2, & \text { if } x>0 \\ 2, & \text { if } x=0\end{cases}
$$

where $\gamma \in(0,1)$, satisfies the condition of Theorem 1 with $\bar{x}_{n}=\frac{1}{4 n-3} \frac{2}{\pi}, \underline{x}_{n}=\frac{1}{4 n-1} \frac{2}{\pi}, n \in$ $\mathbb{N}$, using $\alpha=2(2 \pi)^{-\gamma}$. Figure 1 illustrates the behavior of $f$ and of the corresponding $g$ and Riemann-Stieltjes integral, as defined in the proof of Theorem 1. 


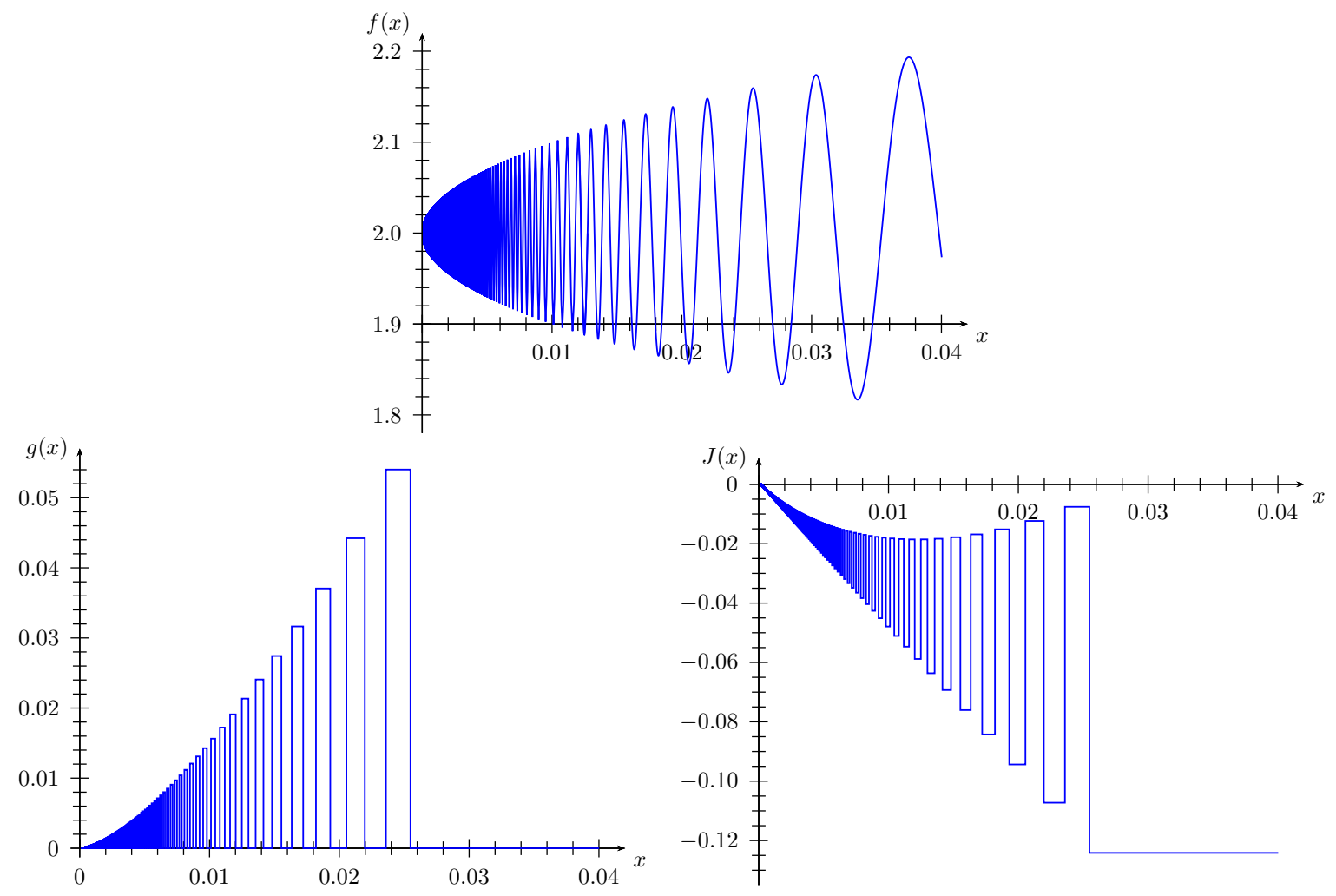

Figure 1. Plot of $f$, as defined in Example 1 for $\gamma=\frac{1}{2}$, and the corresponding functions $g$ and $J, J(x):=\int_{0}^{x} f\left(x^{\prime}\right) \mathrm{d} g\left(x^{\prime}\right)$, as defined in the proof of Theorem 1 using $\beta=\frac{3}{2}$ and $n_{0}=7$. The values have been computed numerically by considering only contributions with $n \leqslant 1000$. For clarity, only the region with $x \in[0,0.04]$ is shown.

\section{INTEGRANDS OF BOUNDED VARIATION}

Any integrand $f$ that satisfies the condition of Theorem 1 is clearly of unbounded variation. This prompts us to ask, could we actually obtain an affirmative answer to Question 1 if $f$ varied "less." In fact, we are able to show that, if $f$ is of bounded variation, the answer to Question 1 is yes. Bounds for Riemann-Stieltjes integrals under these assumptions have been derived by Beesack [1], Ganelius [3], and Knowles [6], but instead of building our argument on them, we give a direct proof which relies on some elementary measure theory and the measure-theoretic version of Grönwall's inequality.

Theorem 2. Let $f:[a, b] \rightarrow(0, \infty)$ be a continuous function and $g:[a, b] \rightarrow[0, \infty)$ a non-vanishing function of bounded variation such that $g(a)=0$. If $f$ is of bounded variation, then

$$
\int_{a}^{y} f(x) \mathrm{d} g(x)>0 \quad \text { for some } y \in(a, b] \text {. }
$$


Proof. Let us denote by $V_{c}^{d} f$ the total variation of $f$ on $[c, d] \subset[a, b]$. Recall that total variation is additive in the sense that $V_{a}^{d} f=V_{a}^{c} f+V_{c}^{d} f$ for $a \leqslant c \leqslant d[7$, Theorem 12.1]. Moreover, since $f$ is continuous, the mapping $x \mapsto V_{a}^{x} f$ from $[a, b]$ to $[0, \infty)$ is continuous [7, Theorem 12.2]. Thus, there exists a finite, positive Borel measure $\nu$ on $[a, b]$ such that $\nu([c, d))=V_{c}^{d} f$ for any $c$ and $d$ such that $a \leqslant c<d \leqslant b$. Let us define another finite, positive Borel measure by $\mu(\mathrm{d} x):=f(x)^{-1} \nu(\mathrm{d} x)$. By construction, we have then

$$
|f(d)-f(c)| \leqslant \int_{[c, d)} f(x) \mu(\mathrm{d} x) .
$$

By an approximation with suitable Riemann-Stieltjes sums, where the values of $g$ are chosen to be sufficiently close to their respective infima on all subintervals of the partitions, we can prove that

$$
\left|\int_{a}^{y} g(x) \mathrm{d} f(x)\right| \leqslant \int_{[a, y)} f(x) g(x) \mu(\mathrm{d} x) .
$$

The Riemann-Stieltjes integral on the left hand side is well-defined by the integration by parts formula [7, Theorem 12.14]

$$
\int_{a}^{y} g(x) \mathrm{d} f(x)=f(y) g(y)-f(a) g(a)-\int_{a}^{y} f(x) \mathrm{d} g(x),
$$

whenever $\int_{a}^{y} f(x) \mathrm{d} g(x)$ exists and this is always true under the present assumptions.

Now suppose that, contrary to our assertion, we have

$$
\int_{a}^{y} f(x) \mathrm{d} g(x) \leqslant 0 \quad \text { for all } y \in(a, b] .
$$

Rearranging the integration by parts formula (4) and using the assumption $g(a)=0$, (3), and (5), we obtain for any $y \in(a, b]$

$$
f(y) g(y) \leqslant \int_{a}^{y} g(x) \mathrm{d} f(x) \leqslant \int_{[a, y)} f(x) g(x) \mu(\mathrm{d} x) .
$$

But the measure-theoretic version of Grönwall's inequality [2, Theorem A.5.1] implies that then $f(x) g(x) \leqslant 0$ for all $x \in[a, b]$, whence $g=0$, a contradiction.

Remark 2. There are two straightforward refinements to Theorem 2. Firstly, it clearly suffices that $f$ is of bounded variation on $\left[x_{\mathrm{L}}, x_{\mathrm{L}}+\varepsilon\right]$ for some $\varepsilon>0$. Secondly, if $g$ is right-continuous, then also the mapping

$$
y \mapsto \int_{a}^{y} f(x) \mathrm{d} g(x)
$$

is right-continuous and, under the assumptions of Theorem 2, there exists an interval $[c, d] \subset[a, b]$ such that

$$
\int_{a}^{y} f(x) \mathrm{d} g(x)>0 \quad \text { for all } y \in[c, d] .
$$


Remark 3. Theorem 2 explains why the answer to Question 1 is perhaps elusive. Experimentation with nicely behaving integrands will not suffice, since a "pathological" $f$ is required in order to discover the general answer. However, such integrands need not be mere curiosities. In fact, our study of Question 1 was originally motivated by an application in financial mathematics involving as the integrand a path of a continuous-time stochastic process, which is typically of unbounded variation.

\section{ACKNOWLEDGEMENTS}

This version corrects a mistake in the formulation of Theorem 1, pointed out to us by Gerald Teschl. J. Lukkarinen was supported by the Academy of Finland. M. S. Pakkanen acknowledges support from the Finnish Cultural Foundation, from CREATES, funded by the Danish National Research Foundation, and from the Aarhus University Research Foundation regarding the project "Stochastic and Econometric Analysis of Commodity Markets".

\section{REFERENCES}

[1] P. R. Beesack, Bounds for Riemann-Stieltjes integrals, Rocky Mountain J. Math. 5 (1975) 75-78. Available at http://dx.doi.org/10.1216/RMJ-1975-5-1-75.

[2] S. N. Ethier and T. G. Kurtz, Markov Processes: Characterization and Convergence, Wiley, New York, 1986.

[3] T. Ganelius, Un théorème taubérien pour la transformation de Laplace, C. R. Acad. Sci. Paris 242 (1956) 719-721.

[4] E. Hewitt and K. Stromberg, Real and Abstract Analysis, Springer, New York, 1965.

[5] T. H. Hildebrandt, Introduction to the Theory of Integration, Academic Press, New York, 1963.

[6] I. Knowles, Integral mean value theorems and the Ganelius inequality, Proc. Roy. Soc. Edinburgh Sect. A 97 (1984) 145-150. Available at http://dx.doi.org/10.1017/S0308210500031917.

[7] M. H. Protter and C. B. Morrey, A First Course in Real Analysis, Springer, New York, 1977.

[8] W. Rudin, Principles of Mathematical Analysis, McGraw-Hill, New York, 1953.

[9] U. V. Satyanarayana, A note on Riemann-Stieltjes integrals, Amer. Math. Monthly 87 (1980) 477-478. Available at http://www.jstor.org/stable/2320259.

Jani Lukkarinen, Department of Mathematics and Statistics, University of Helsinki, P.O. Box 68, FI-00014 Helsingin yliopisto, Finland.

E-mail address: jani.lukkarinen@helsinki.fi

Mikko S. Pakkanen, CREATES and Department of Economics and Business, Aarhus

University, Fuglesangs Allé 4, DK-8210 Aarhus V, Denmark.

E-mail address: msp@iki.fi

URL: http://www.mikkopakkanen.fi/ 